\title{
Determination of Rare Earth Elements and Yttrium in Geological Materials by Inductively Coupled Plasma Atomic Emission Spectrometry Using Cu-DDTC as Coprecipitative Carrier
}

\author{
M. Krishnakumar ${ }^{\text {** }}$ and Manjeet Kumar ${ }^{\mathrm{b}}$ \\ ${ }^{a}$ Chemistry Laboratory, Atomic Minerals Directorate for Exploration and Research, \\ Department of Atomic Energy, Begumpet, Hyderabad-500629, India \\ ${ }^{b}$ Chemistry Laboratory, Atomic Minerals Directorate for Exploration and Research, \\ Department of Atomic Energy, R. K. Puram, West Block VI, New Delhi-110066, India
}

\section{INTRODUCTION}

Rare earth elements (REEs) and yttrium (Y) find varied applications in different fields such as for lasers, phosphors, magnetic bubble memory films, fiber optics, superconductors, and nuclear reactor materials. REEs constitute one of the most important groups of trace elements in geochemical studies. The degree of REE fractionation in rocks or minerals acts as petrogenetic indicator to understand the evolution of igneous rocks which involves processes such as partial melting of crustal or mantle materials, fractional crystallization, and/or mixing of magmas (1-3). Thus simple, rapid, and accurate methods for REE and Y determination are needed to handle large quantities of samples taken up during a geochemical survey.

Neutron activation analysis (NAA) and isotope dilution mass spectrometry (IDMS) are the principal reference techniques to determine traces of REEs in geological samples (4). These methods are time-consuming and involve complicated experimental procedures at nuclear reactor facilities. Inductively coupled plasma mass spectrometry (ICP-MS) is a highly sensitive technique for REE determination. However, these instruments are very expensive, and the technique suffers from isobaric spectral interferences.

*Corressponding autbor

E-mail: kkatdae@gmail.com

\section{ABSTRACT}

A rapid, simple, and precise method for the determination of trace rare earth elements (REEs) and $\mathrm{Y}$ in complex geological samples by inductively coupled plasma atomic emission spectrometry (ICP-AES) is described. The REEs and $\mathrm{Y}$ as a group were separated and preconcentrated using copper diethyldithiocarbamate $(\mathrm{Cu}-$ DDTC) as coprecipitative carrier. Variables such as the optimum $\mathrm{pH}$, amount of carrier concentration, and quantity of DDTC were optimized for the quantitative recovery of the analyte of interest. Using the proposed procedure, the REEs and $\mathrm{Y}$ were separated from $\mathrm{Ca}, \mathrm{Mg}, \mathrm{Ba}, \mathrm{Cr}$, $\mathrm{Mo}, \mathrm{Nb}$, and $\mathrm{Ta}$. The method was validated by analyzing standard reference materials AC-E Granite and SY-4 Syenite. The method was found to be precise with a RSD $\leq 10 \%$. The developed method was applied to the determination of REEs and $\mathrm{Y}$ in complex geological samples having varying matrices.

Inductively coupled plasma atomic emission spectrometry (ICP-AES) is the commonly used technique for the determination of trace REEs . It offers multi-element detection capabilities over a wide concentration range with relatively low detection limits, high levels of precision and accuracy, rapid analytical procedures, and excellent calibration linearity (5). The technique is also free from chemical interferences. However, it is generally recognized that a substantial part of the detection power of ICPAES may be lost if the sample contains major elements with rich line spectra. Elements such as uranium, barium, zirconium, etc., interfere seriously in the determination of REEs. Moreover, REEs are found at trace to ultra-trace levels in geological samples and since most of the matrix elements interfere with REE determination owing to the spectral overlaps and background continuum interferences (6), a prior separation and preconcentration from the rest of the matrix is essential. This further increases the sensitivity of the technique.

A number of separation and preconcentration methods have been successfully applied in the group separation and determination of REEs in various alloys, rocks, and geological materials (7). Liquidliquid extraction utilizes preferable distribution of the analyte from the aqueous phase to the organic phase. However, this requires large quantities of hazardous and costly organic solvents. Methods involving chelating resins and solid phase extraction procedures are selective to the analytes of interest but are time-consuming and require sophisticated glassware as well as desorption of the elements from the solid support. On-line methods such as flow injection analysis offer freedom from contamination $(8,9)$. Co-precipitation is the simplest and also a fast preconcentration procedure. This allows the quantitative precipitation of the metal ion of 
interest by the addition of a co-precipitant. Since many matrix elements co-precipitate along with the analytes, this process can be minimized by selecting suitable conditions during precipitation and subsequent washing of the resulting precipitate. The effect of interfering elements can be minimized by measuring the emission intensity at selected wavelengths.

Sodium diethyldithiocarbamate, $\left(\mathrm{C}_{5} \mathrm{H}_{10} \mathrm{NS}_{2} \mathrm{Na}\right), \mathrm{Na}-\mathrm{DDTC}$, is a known chelating agent for many metal ions, especially heavy metals and mercury. The reagent is readily soluble in water but much less soluble in organic solvents and is generally used with masking agents like EDTA and KCN, to enhance its selectivity at a particular $\mathrm{pH}$. However, the compounds of metals with DDTC are insoluble in water since they are uncharged. The complexes decompose readily under highly acidic conditions into diethylamine and carbon disulphide leaving the analyte of interest free for analysis by a suitable technique (10). Na-DDTC was used in the separation and ICP-AES determination of trace elements including $\mathrm{Zr}$ and Hf in water samples (11). Na-DDTC has also been used in the determination of elements such as As(III), $\mathrm{Bi}, \mathrm{Sb}(\mathrm{III}) \mathrm{Se}(\mathrm{IV}), \mathrm{Te}(\mathrm{IV}), \mathrm{Tl}(\mathrm{III})$, and V(V).

The present investigation reports the determination of REEs and $\mathrm{Y}$ in geological materials by ICP-AES after a simple and fast coprecipitative separation and preconcentration of these elements as a group with copper diethyldithiocarbamate. The method was validated with the analysis of two international standard reference materials: AC-E Granite and SY-4 Syenite. The same method was also applied to the analysis of geological samples collected during a uranium exploration. The analytical results were compared with the values obtained by the well-established activated charcoal separation of
REEs and $\mathrm{Y}$ as their fluorides and subsequent determination by ICPAES (14).

\section{EXPERIMENTAL}

\section{Instrumental}

All emission intensity measurements were performed using the Model Ultima2 ICP-AES, radial view (Horiba Jobin Yvon, France), equipped with a crystal-controlled RF generator of $40.68 \mathrm{MHz}$ and a monochromator (focal length $1 \mathrm{~m}$ ), employing ruled back-to-back grating of 4320 and 2400 grooves $/ \mathrm{mm}$. The sample introduction system had a concentric glass nebulizer and cyclonic spray chamber incorporated with a sheath gas device. Background corrections were applied wherever necessary. The instrumental parameters used are listed in Table I. All pH adjustments were made using a digital $\mathrm{pH}$ meter consisting of a glass electrode with an automatic temperature control (Systronics, India).

\section{Reagents and Standard Solutions}

The reagents used for the preparation of the samples and standards were of AR/GR grade. All singleelement REEs and Y (as oxides) of $1 \mathrm{mg} / \mathrm{mL}$ standard stock solutions were made by dissolving SpecPure ${ }^{\circledR}$ rare earth oxides (Johnson and Matthey, UK) in nitric acid, except for $\mathrm{CeO}_{2}$ which was dissolved in a mixture of $\mathrm{HNO}_{3}$ and $\mathrm{H}_{2} \mathrm{O}_{2}$.

Multi-element calibration standard solutions were prepared by dilution of the stock solutions with Milli-Q ${ }^{\mathrm{TM}}$ water (Millipore Corporation, Bedford, MA, USA), keeping the final acidity at $3 \%(\mathrm{v} / \mathrm{v}) \mathrm{HNO}_{3}$. Na-DDTC (Fisher Scientific, India) was used as received. The copper solution $(10 \mathrm{mg} / \mathrm{mL})$ was prepared by dissolving pure metal in subboiled $\mathrm{HNO}_{3}$.

\section{Sample Pretreatment \\ Sample Decomposition}

Geological samples (1.0 g) were treated with $5 \mathrm{~mL}$ of $\mathrm{HF}$ (40\%) and $3 \mathrm{~mL} \mathrm{HNO}_{3}$. The evaporation process was repeated three times. Then the contents were dissolved in $20 \mathrm{~mL}$ of $1: 1 \mathrm{HNO}_{3}$ and transferred to a glass beaker.

\section{Co-precipitation}

To the above solution, $2 \mathrm{mg} \mathrm{Cu}$ was added and the $\mathrm{pH}$ adjusted to 6 with the digital $\mathrm{pH}$ meter using dilute ammonia/nitric acid solutions. Then $50 \mathrm{mg}$ of crystalline sodium diethyldithiocarbamate (Na-DDTC) was added while stirring. This solution was kept standing for $10 \mathrm{~min}$ utes with continuous stirring. The dark brown precipitate of Cu-DDTC loaded with the analytes was filtered through a Whatman 541 filter paper. After washing the pre-

TABLE I

Instrumental Specifications and Operating Conditions of ICP-AES

\begin{tabular}{ll}
\hline Parameters & Specifications \\
\hline Monochromator Mounting & Czerny-Turner \\
Order of Measurement & First order \\
First Order Resolution & \\
$(180-420 \mathrm{~nm})$ & $0.005 \mathrm{~nm}$ \\
Power Applied & $1 \mathrm{~kW}$ \\
Plasma Gas & Argon \\
Plasma Gas Flow Rate & $12 \mathrm{~L} / \mathrm{min}$ \\
Sheath Gas Flow Rate & $0.2 \mathrm{~L} / \mathrm{min}$ \\
Nebulizer Gas Flow Rate & $0.5 \mathrm{~L} / \mathrm{min}$ \\
\hline
\end{tabular}


cipitate thoroughly with distilled water, it was carefully transferred to a beaker with a fine jet of water. The filter paper was also rinsed with a few drops of $\mathrm{HNO}_{3}$ to ensure the complete transfer of the precipitate. The precipitate was dissolved in 2-3 $\mathrm{mL}$ of $\mathrm{HNO}_{3}$ by boiling thoroughly and then evaporated to incipient dryness. The clear solution was diluted to $25 \mathrm{~mL}$ final volume after maintaining 5\% (v/v) acidity with nitric acid. However, it was found that the above precipitate, if needed, can be made up to $10 \mathrm{~mL}$ final volume. The elemental determinations were carried out by aspirating the solution into the ICP-AES at the selected wavelengths with minimum inter-elemental interferences.

\section{RESULTS AND DISCUSSION}

Preliminary experiments were started with co-precipitation of europium and its determination by flame atomic absorption spectrometry (FAAS). The results obtained were encouraging. Thus a synthetic mixture of REEs (La-Lu, Y) was pre- pared, and the above procedure applied by separating the elements and analyzing them by ICP-AES at the specified wavelengths. The results are presented in Table II.

\section{Effect of pH on Co-precipitation}

The $\mathrm{pH}$ for co-precipitation was optimized by varying it from 1-10. However, it was observed that the the recovery was negligible below pH 5 for all elements. However, quantitative recovery of all elements was obtained from $\mathrm{pH} 6$ onwards. Hence, the $\mathrm{pH}$ of 6 was chosen for all further studies. Coprecipitation of the metal ions on the collectors can be attributed to several mechanisms such as adsorption, where the charge on the surface can attract ions in solution of opposite charge or through occlusion during which the ions are embedded within the precipitate formed. Co-crystallization of the analyte along with the carrier precipitate has also been suggested as the mechanism for co-precipitation (12). The major disadvantage of coprecipitation is that the precipitate,

TABLE II

Analytical Results Obtained for Synthetic Mixture of REEs and Y (wavelengths given in parentheses)

\begin{tabular}{ccccc}
\hline $\begin{array}{c}\text { Element } \\
\text { (Wavelength: } \mathrm{nm})\end{array}$ & $\begin{array}{c}\text { Added } \\
(\mu \mathrm{g} / \mathrm{mL})\end{array}$ & $\begin{array}{c}\text { Found } \\
(\mu \mathrm{g} / \mathrm{mL})\end{array}$ & $\begin{array}{c}(\%) \\
\text { Recovery }\end{array}$ & $\begin{array}{c}\text { RSD } \\
(\%)\end{array}$ \\
\hline La (333.749) & 0.80 & 0.78 & 98 & 8 \\
Ce (418.660) & 0.64 & 0.60 & 94 & 8 \\
Pr (422.293) & 0.80 & 0.74 & 93 & 10 \\
Nd (430.357) & 2.00 & 1.81 & 91 & 7 \\
Sm (359.260) & 0.80 & 0.74 & 93 & 8 \\
Eu (381.966) & 0.08 & 0.08 & 100 & 5 \\
Gd (342.247) & 0.40 & 0.38 & 95 & 6 \\
Tb (350.917) & 0.40 & 0.38 & 95 & 9 \\
Dy (353.170) & 0.20 & 0.19 & 95 & 8 \\
Ho (345.600) & 0.08 & 0.08 & 100 & 9 \\
Er (349.910) & 0.50 & 0.47 & 94 & 9 \\
Tm (346.220) & 0.20 & 0.19 & 95 & 9 \\
Yb (328.937) & 0.04 & 0.04 & 100 & 6 \\
Lu (261.542) & 0.02 & 0.02 & 100 & 8 \\
Y (371.029) & 0.08 & 0.08 & 100 & 5 \\
\hline
\end{tabular}


tion could be of use for determining trace REEs in normal geological matrices. Trace elements like $\mathrm{Ba}$, $\mathrm{Cd}, \mathrm{Co}, \mathrm{Cr}, \mathrm{Mo}, \mathrm{Ni}, \mathrm{Nb}, \mathrm{Pb}, \mathrm{Sr}$, Ta, $\mathrm{V}, \mathrm{Zn}$, and $\mathrm{Zr}$ at the concentration level of $10 \mu \mathrm{g} / \mathrm{mL}$ were added to a synthetic mixture of REEs $(1 \mu \mathrm{g} / \mathrm{mL})$. No quantifiable interference was observed at the wavelengths used. Further, $\mathrm{Ba}, \mathrm{Sr}, \mathrm{Mo}, \mathrm{Nb}, \mathrm{Ta}$, and $\mathrm{Cr}$ were not co-precipitated. It can be seen that these results further enhance the utility of the proposed method in the determination of REEs and $\mathrm{Y}$ even at trace levels in matrices rich in the above elements.

\section{Method Validation}

A set of two certified reference materials, AC-E (Granite, Centre
National De La Recherche Scientifique, France) and SY-4 (Syenite, CANMET, Canada), containing sizable amounts of the analytes of interest, were selected and analyzed for evaluation of the effectiveness of the proposed methodology. Table III lists the values obtained with the present method which are in good agreement with the certified values.

\section{Application to Real Geological Samples}

The proposed method (PM) was also applied to the ICP-AES determination of REEs and $\mathrm{Y}$ in several rock samples received during an exploration for atomic minerals. The data from this analysis were compared with the values obtained by separation and preconcentration of these elements as their fluorides on activated charcoal (14). The results of the present method (PM) and the fluoride precipitation-activated charcoal method (FPM) are listed in Table IV. The entire process of the proposed co-precipitation and separation method takes less than one hour for a batch of 10 samples which includes the time required for preparation of the standards for calibration. Hence, the present method is rapid compared to other rare earth element separation methods such as solvent extraction and solid phase extraction which are generally and routinely adopted.

TABLE III

Comparison of Results Obtained for CRMs, AC-E (Granite) \& SY-4 (Syenite) (values in $\mu \mathrm{g} / \mathrm{g}$ )

\begin{tabular}{|c|c|c|c|c|c|c|c|c|c|c|c|c|c|c|c|c|}
\hline CRM & Comparison & $\mathrm{La}$ & $\mathrm{Ce}$ & $\operatorname{Pr}$ & $\mathrm{Nd}$ & Sm & $\mathrm{Eu}$ & Gd & $\mathrm{Tb}$ & Dy & Ho & $\mathrm{Er}$ & $\mathrm{Tm}$ & $\mathrm{Yb}$ & $\mathrm{Lu}$ & $\mathrm{Y}$ \\
\hline \multicolumn{17}{|c|}{ AC-E } \\
\hline & Value Obtained & 56 & 150 & 19 & 85 & 22 & 1.9 & 24 & 5 & 30 & 6 & 16 & 2 & 16.4 & 2.4 & 190 \\
\hline & Certified Value & 59 & 154 & 22.2 & 92 & 24.2 & 2 & 26 & 4.8 & 29 & 6.5 & 17.7 & 2.6 & 17.4 & 2.4 & 184 \\
\hline \multicolumn{17}{|c|}{ SY-4 } \\
\hline & Value Obtained & 56 & 119 & 13 & 53 & 11 & 1.9 & 13 & $<3$ & 18 & 4 & 13 & 2.0 & 13.9 & 2.0 & 120 \\
\hline & Certified Value & 58 & 122 & 15 & 57 & 12.7 & 2 & 14 & 2.6 & 18.2 & 4.3 & 14.2 & 2.3 & 14.8 & 2.1 & 119 \\
\hline
\end{tabular}

TABLE IV

Comparison of Results Obtained for REEs and Y in Geological Samples by Proposed Method (PM) and Fluoride Precipitation-Activated Charcoal Method (FPM) (values in $\mu$ g/g)

\begin{tabular}{|c|c|c|c|c|c|c|c|c|c|c|c|c|c|c|c|}
\hline Sample Comparison & La & $\mathrm{Ce}$ & $\operatorname{Pr}$ & $\mathrm{Nd}$ & $\mathrm{Sm}$ & $\mathrm{Eu}$ & Gd & $\mathrm{Tb}$ & Dy & Ho & $\mathrm{Er}$ & $\mathrm{Tm}$ & $\mathrm{Yb}$ & $\mathrm{Lu}$ & $\mathrm{Y}$ \\
\hline \multicolumn{16}{|l|}{ AMD-1 } \\
\hline P.M. & 100 & 280 & 30 & 110 & 20 & 1.0 & 20 & 4 & 14 & 3 & 4 & $<1$ & 3.1 & $<1.0$ & 89 \\
\hline F.P.MO & 115 & 290 & 35 & 130 & 26 & 1.1 & 23 & 4 & 15 & 3 & 5 & $<1$ & 3.4 & $<1.0$ & 81 \\
\hline \multicolumn{16}{|l|}{ AMD-2 } \\
\hline P.M. & 35 & 90 & $<10$ & 39 & $<10$ & $<1$ & 9 & $<3$ & 12 & 3 & 6 & $<1$ & 6.0 & $<1.0$ & 90 \\
\hline F.P.M & 37 & 97 & 12 & 43 & $<10$ & $<1$ & 10 & $<3$ & 13 & 3 & 8 & $<1$ & 7.1 & $<1.0$ & 91 \\
\hline \multicolumn{16}{|l|}{ AMD-3 } \\
\hline P.M. & 30 & 72 & $<10$ & 30 & $<10$ & 1.1 & $<5$ & $<3$ & 4 & $<3$ & $<3$ & $<1$ & 2.5 & $<1.0$ & 38 \\
\hline F.P.M & 32 & 80 & $<10$ & 35 & $<10$ & 1.2 & 5 & $<3$ & 5 & $<3$ & 3 & $<1$ & 3.0 & $<1.0$ & 36 \\
\hline
\end{tabular}




\section{Atomic \\ 1 Vol. 32(6), Nov./Dec. 2011}

\section{CONCLUSION}

The proposed procedure using copper diethyldithiocarbamate $(\mathrm{Cu}-$ DDTC) co-precipitation, separation, and preconcentration is simple, rapid, and accurate for the determination of rare earth elements (REEs) and yttrium (Y) in geological samples by inductively coupled plasma atomic emission spectrometry (ICPAES).

The procedure is precise with a $\leq 10 \%$ RSD for triplicate determinations of various rare earth elements and yttrium. This method can be applied on a routine basis for the determination of trace REEs and $\mathrm{Y}$ in samples with complex matrices and especially rich in $\mathrm{Ca}$, $\mathrm{Mg}, \mathrm{Ba}, \mathrm{Mo}, \mathrm{Cr}, \mathrm{Nb}$, and Ta since the preconcentration procedure excludes these elements. The method does not require large quantities of organic solvents, thus is environmentally friendly, and demonstrates the significance and effectiveness of coprecipitation.

\section{ACKNOWLEDGMENTS}

The authors wish to thank Dr. K. Satyanarayana, Head, Chemistry Group, AMD. for his constant support of the research and development work. We are grateful to the Additional Director (R\&D), AMD, for constant encouragement and to the Director, AMD for granting kind permission to publish this work.

Received July 20, 2011.

\section{REFERENCES}

1. P. Henderson (ed.), Rare Earth Element Geochemistry, Elsevier, Amsterdam, The Netherlands (1984).

2. K. Satyanarayana, At. Spectrosc. 17, 69 (1996).

3. M. S. Navaro, H. H. G. J. Ulbrich, S. Andrade, and V. A. Janasi, J. Alloys and Compounds 344, 40 (2002).

4. I. Roelandt, Chem. Geol. 67, 171 (1988).

5. P. K. Srivastava, and A. Premadas, J. Anal. At. Spectrom. 14, 1087 (1999).

6. D. W. Zachmann, Anal. Chem. 60, 420 (1988).

7. J. N. Walsh, F. Buckley, and J. Barker, Chem. Geol. 33, 141 (1981).

8. M. Kumar, D. P. S. Rathore, and A. K. Singh, Analyst 125, 1221 (2000).

9. T. Prasada Rao, and J. M. Gladis, Anal. Sci. 18, 517 (2002).

10. J. Minczewski, J. Chwastowska, and R. Dybezynski, "Separation and Preconcentration Methods in Inorganic Analysis" 209-212, Ellis Horwood Limited, Chichester, UK (1982).

11. R. Bose, and K. Satyanarayana, EARFAM 19, 72 (2009).

12. John R. Dean "Practical Inductively Coupled Plasma Spectroscopy" 8788, John Wiley \& Sons Ltd., England (1999).

13. O. Fujino, S. Umetani, E. Ueno, K. Shigeta, and T. Matsuda, Anal. Chim. Acta 420, 65 (2000).

14. D. S. R. Murthy, P. L. Mahanta and R. Radhamani, At. Spectrosc. 23(2), 65 (2002) 